\title{
Increased Intestinal Permeability and Parkinson Disease Patients: Chicken or Egg?
}

\author{
D. Salat-Foix, K. Tran, R. Ranawaya, J. Meddings, O. Suchowersky
}

\begin{abstract}
Gastrointestinal involvement is a frequent and early event in the course of Parkinson Disease (PD), and may have a prominent role in the early pathophysiology of the disease. On the other hand, derangement in intestinal permeability could also result from the involvement of the gastrointestinal tract over the course of the disease. Patients and methods: The intestinal permeability of 12 non-selected PD patients was studied using a validated, non-invasive test; these results were compared to predefined age-adjusted reference values. Results: 4/12 PD patients had abnormal gastrointestinal permeability; two had both an abnormal lactulose/mannitol ratio and an abnormal sucrose concentration, and two an isolated abnormal result. An increased lactulose/mannitol ratio is consistent with defect of either the enterocytes or the tight junctions between them. Conclusion: Intestinal permeability is increased in a significant proportion of unselected PD patients with minimal gastrointestinal symptoms. The significance of this finding needs to be further evaluated.
\end{abstract}

RÉSUMÉ: Augmentation de la perméabilité intestinale chez les patients atteints de la maladie de Parkinson : la poule ou l'œuf ? L'atteinte gastro-intestinale est fréquente et précoce dans l'évolution de la maladie de Parkinson (MP) et pourrait jouer un rôle important tôt dans la physiopathologie de la maladie. D'autre part, l'altération de la perméabilité intestinale pourrait également être due à une atteinte du tractus gastrointestinal au cours de l'évolution de la maladie. Méthode : Nous avons étudié la perméabilité intestinale de 12 patients atteints de la MP, choisis au hasard, au moyen d'un test validé non effractif. Nous avons comparé leurs résultats à des valeurs de référence préétablies ajustées pour l'âge. Résultats : La perméabilité gastro-intestinale était anormale chez 4 des 12 patients. Deux avaient un rapport lactulose/mannitol anormal et une concentration de sucrose anormale et 2 avaient un résultat anormal isolé. Une augmentation du rapport lactulose/mannitol est en faveur d'un défaut soit des entérocytes ou des jonctions serrées entre eux. Conclusion : La perméabilité intestinale est augmentée chez une proportion importante de patients atteints de la MP, choisis au hasard, qui ont peu de symptômes gastro-intestinaux. La signification de cette observation mérite d'être étudiée davantage.

Can J Neurol Sci. 2012; 39: 185-188

Gastrointestinal involvement occurs early in the course of Parkinson Disease (PD), as shown both by clinical ${ }^{1-3}$ and pathological ${ }^{4,5}$ studies. These findings have led to speculations about the role of gastrointestinal dysfunction in the early pathophysiology of the disease $e^{6,7}$. It has been conjectured that an ingested neurotoxin could permeate the gastrointestinal mucosa, reach the enteric nervous system, locally induce the aggregation of native $\alpha$-synuclein, trigger cell death, and subsequently reach the dorsal motor of the vagus nerve via retrograde axonal transport, and thus be the primary pathological event in the development of PD. On the other hand, it has been well established that the frequency and severity of gastrointestinal symptoms tend to increase with disease progression ${ }^{8}$, thus suggesting that the involvement of the gastrointestinal tract may be a dynamic process over the course of the disease.

Given the high prevalence of gastrointestinal complaints among PD patients (reported by as high as $88 \%$ in some existing reviews ${ }^{8}$ ) we decided to systematically evaluate 15 consecutive early-to-moderate PD patients seen at our Clinic, both from a neurological and a gastrointestinal perspective. Among the multiple tests of gastrointestinal function, we decided to focus on mucosal permeability. Derangement in gastrointestinal permeability may be significantly involved in the pathogenesis of $\mathrm{PD}^{6}$, and, although its presence cannot be inferred from any given gastrointestinal complaint, it can be easily studied using a standardized, validated, non-invasive and relatively inexpensive test.

The foundations of intestinal permeability studies have been discussed elsewhere ${ }^{9}$. In short, small, nontoxic, noncharged, water soluble compounds that will be largely excreted in the urine without being metabolized if absorbed from the gastrointestinal tract are given to the study subjects. Sucrose (to test gastro-duodenal absorption), lactulose and mannitol (which test absorption in more distal segments of the small intestine) are commonly used. The urinary concentration of the compounds is directly proportional to their degree of absorption in the gastrointestinal tract. By comparing these values to previously determined reference intervals, it is inferred whether a subject's intestinal permeability is abnormal.

From the Movement Disorders Program, Department of Clinical Neurosciences (DSF, RR), Division of Gastroenterology, Department of Internal Medicine (KT, JM),

University of Calgary, Calgary; Department of Medicine (Neurology) and Medical Genetics (OS), University of Alberta, Edmonton, Alberta, Canada.

Received August 29, 2011. Final Revisions Submitted October 19, 2011.

Correspondence to: David Salat-Foix, Movement Disorders Program. Department of

Clinical Neurosciences, Area 3, UCMC, 3350 Hospital Drive NW, Calgary, Alberta,

T2N 4N1, Canada. 


\section{Patients And Methods}

Considering that we had not defined stringent selection criteria and that individuals in the study were not stratified into groups, this study can be considered to be observational. On top of this, all of the procedures in the study's protocol are routinely employed either in the Movement Disorders or the Gastrointestinal Clinic. For these reasons, the implicit consent that pertains to the standard clinical practice was considered to be operative in this setting, and no further approval by the Ethics Board was sought.

Consecutive early-to-moderate PD patients diagnosed according to the United Kingdom's Parkinson's Society Brain Bank Clinical Diagnostic Criteria ${ }^{10}$ were invited to participate in the study. Those with a prior diagnosis of celiac disease, inflammatory bowel disease, irritable bowel syndrome, seronegative spondyloarthropaty, primary biliary cirrhosis, cachexia, HIV disease, acute pancreatitis, chronic heart failure, type 1 diabetes mellitus, or nonalcoholic fatty liver disease were excluded. In total, the clinic records of the fifteen patients were reviewed, and a standard visit covering their current symptoms (both motor and non-motor) and the response to therapy was performed. At the end of the visit a kit and appropriate instructions to perform the gastrointestinal permeability test were given to the patients.

Information on age and gender, times since onset of motor symptoms and PD diagnosis, the presence and nature of prodromal gastrointestinal symptoms, and current medications for both PD and gastrointestinal complaints was collected. A levodopa equivalent daily dose (LEDD) was calculated by applying the following corrections: sustained release Ldopax0.75, amantadine dosex 1, L-dopa with a catechol-Omethyl transferase-inhibitor dose of L-dopax1.3, pramipexole and rasagiline dosex $100^{11}$. The NMS-Quest ${ }^{12}$ and the MDSUPDRS part III ${ }^{13}$ were subsequently scored.

Study participants were instructed to stop the ingestion of any alcoholic beverage at least five days prior to performing the test. On the day they chose to perform it, they were allowed to eat and drink freely until $6 \mathrm{pm}$, and asked to empty their bladder and take a test solution (a flavored mixture of $100 \mathrm{~g}$ sucrose, $5 \mathrm{~g}$ lactulose and $2 \mathrm{~g}$ mannitol reconstituted in $450 \mathrm{~mL}$ of water) before going to bed. The overnight urine output was collected for analysis.

Samples were kept refrigerated until analyzed by highpressure liquid chromatography. Chromatography refers to the various techniques by which a certain compound can be detected and quantified in a problem solution. When a fluid flows along thin spaces between densely packed solid columns, the interaction between its molecules and those at the surface of the columns (a process determined largely by the size and polarity of the flowing molecules) will cause each compound in the fluid to advance at a different speed. High-pressure liquid chromatography relies on this feature for their separation. If the speed at which a compound of interest will move under preset experimental conditions is known, the absorption of ultra-violet (UV) light of a certain wavelength at the time when this substance is expected to move across a detector system can be used to document both its presence and its concentration in the problem solution. The total amount of each one of the probes in the urine was determined, and expressed in mg for sucrose and as a ratio for lactulose and mannitol (lac/man ratio).
Individuals with abnormal lac/man test results were screened for subclinical celiac disease using the $\operatorname{IgA}$ tissue transglutaminase antibody test $\mathrm{t}^{14}$.

Descriptive statistics of our data were expressed as mean and range for quantitative variables, and absolute and relative frequencies for qualitative variables. The reference values for the probes were $<180 \mathrm{mg}$ for sucrose and $<0.025$ for the lac/man ratio.

\section{RESULTS}

Three individuals did not return the urine sample (one argued that her taking medication at bedtime was a violation of the test protocol, one refused to take the test solution as it turned black (due to the flavoring substance that was used) when water was added to the mixture, and one moved out of the province) and they were excluded from the analysis. The other 12 patients, seven men and five women aged 46 to 84 (mean 68.58) years were included in the study. All of these participants reported they had been compliant with the instructions regarding alcohol and food ingestion they had received.

The symptoms that led to the diagnosis of PD had begun on average 82.5 (range 18 to 171 ) months before their participation in the study. All patients receiving dopaminergic treatment were examined while in an on state (mean MDS-UPDRS score 13.64, range 3 to 31); a patient who was not being treated had a MDSUPDRS score of 13. The mean H\&Y scale value was 1.75 (range 1 to 3$)$.

Constipation $(n=4)$ and bloating $(n=1)$ were reported as preceding the diagnosis of PD; nausea/vomiting or abdominal pain were not recalled by any of our patients. Reported symptoms were graded as "long-standing", "mild" and/or "infrequent" by those who had experienced them.

Eleven patients had been treated since the time of diagnosis (mean treatment length 64.45 (range 6 to 144) months). At the time of their inclusion ten patients were taking L-dopa (dose range 300 to $1000 \mathrm{mg}$ ). Six patients were also taking pramipexole ( $\mathrm{n}=3$, dose range 0.5 to $2 \mathrm{mg})$, rasagiline $(\mathrm{n}=1$ in a dose of $1 \mathrm{mg}$ ) or amantadine ( $\mathrm{n}=1$ in a dose of $100 \mathrm{mg})$. One patient was on monotherapy with rasagiline $(1 \mathrm{mg})$. The mean LEDD for treated patients was $556.82 \mathrm{mg}$.

The NMS questionnaire disclosed an average of 7.33 (range 0 to 19 ) non-motor symptoms (1.67 (range 0 to 3 ) of which involving the gastrointestinal tract) per patient. Two patients were taking domperidone (dose of 10 and $20 \mathrm{mg}$ respectively) to relieve gastrointestinal symptoms.

The total sucrose content and the lac/man ratios determined for each of the patients' urine samples are plotted in the Figure. The overall prevalence of gastrointestinal permeability abnormalities was $33.3 \%$, with two patients having both parameters significantly elevated, and two additional patients having an isolated abnormality in one of the tests. The Table shows the distribution of the study participants according to the results of the permeability tests and their self-report of current gastrointestinal symptoms (as per NMS-Quest). An average of 1.25 coexisting gastrointestinal symptoms were reported by participants in the group with normal permeability test results, while patients with abnormal test results reported an average of two symptoms. The celiac screening was negative in all patients with abnormal intestinal permeability results. 


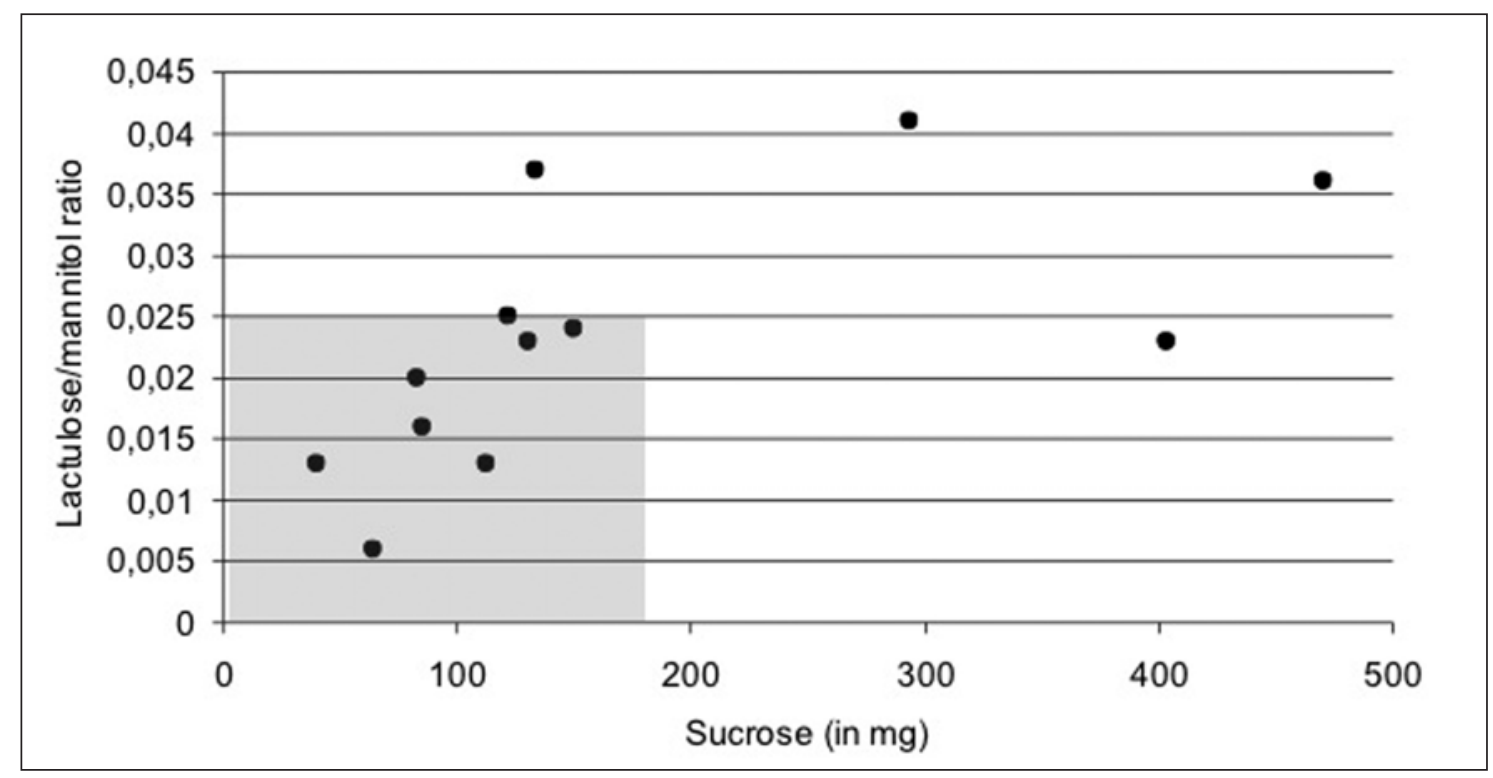

Figure: Results of the gastrointestinal permeability tests for each of the study patients. The shaded area represents normality in the reference population.

\section{DISCUSSION}

Increased intestinal permeability was identified as a significant component of celiac disease in the late 1970 's 15 . Subsequent research found that this abnormality may be significant for the development and progression of various other gastrointestinal disorders, including inflammatory bowel disease and the irritable bowel syndrome $\mathrm{e}^{16,17}$, as well as in systemic disease, such as seronegative spondyloarthropaties ${ }^{18}$, primary biliary cirrhosis, cachexia, the progression of HIV disease, acute pancreatitis, chronic heart failure, type 1 diabetes mellitus, and nonalcoholic fatty liver disease $\mathrm{P}^{19-25}$. Feld et $\mathrm{al}^{19}$ found abnormal permeability test results in $3.9 \%$ of healthy controls $(1.3 \%$ had abnormal sucrose excretion and $4 \%$ an abnormal lac/man ratio, but no subject had an abnormal result on both tests).

Table: Distribution of study participants according to presence of gastrointestinal complaints and permeability test results.

\begin{tabular}{lll}
\hline & $\begin{array}{l}\text { Normal } \\
\text { permeability } \\
\text { tests }\end{array}$ & $\begin{array}{l}\text { Abnormal } \\
\text { permeability } \\
\text { tests }\end{array}$ \\
$\begin{array}{l}\text { No current GI } \\
\text { symptoms }\end{array}$ & $2(2)$ & $0(0)$ \\
$\begin{array}{l}\text { Any current GI } \\
\text { symptom }\end{array}$ & $4(1)$ & $6(9)$ \\
\hline
\end{tabular}

An estimation of expected values based on previously published data is given in parenthesis after the observed values in each cell
The only previous attempt to evaluate intestinal permeability in PD using a differential sugar absorption test was published in 1996 by Davies et $\mathrm{al}^{26}$. As a group, PD patients showed evidence of deranged intestinal permeability (reduced absorption of mannitol and increased lactulose/mannitol ratio) when compared to age and sex matched controls, but individual results in both groups were highly overlapping. As this study was performed at a time when the association between certain systemic conditions and increased intestinal permeability had not been recognized, abnormal results among "healthy controls" may have resulted precisely from the inclusion of individuals with one of these conditions in the control group.

The results from the three patients in our study who had increased intestinal permeability (abnormal lac/man ratio) suggest either epithelial damage or abnormal function at the level of the tight junction in the small intestine. None of the patients in our series were diagnosed with any of the conditions currently thought to relate to abnormal gastrointestinal permeability. The turnover rate for enterocytes has been estimated to be about three days. Since participants in our study had stopped alcohol consumption at least five days before the tests were performed we believe that it is likewise unlikely that the abnormal results can be attributed to an enterotoxic effect of alcohol. In this scenario, our results suggest that the "intestinal barrier function" may be significantly compromised in $25-30 \%$ of PD patients. The cause for these abnormalities and their potential role in the pathogenesis of $\mathrm{PD}$, however, cannot be determined by permeability testing alone.

The isolated abnormality in sucrose excretion found in an additional patient is harder to interpret, as gastric permeability is influenced by multiple factors (such as Helicobacter pylori infection, a stress response, or the ingestion of gastroerosive foods or medications), some of which are not easily controlled for $^{27}$. 
So far, animal models have not been successful in reproducing the pathophysiology of $\mathrm{PD}$, and the enteral administration of rotenone is no exception to this observation ${ }^{28}$. However, Pan-Montojo et $\mathrm{al}^{29}$ have reported that the chronic low-dose exposure of mice to intragastric rotenone induced the sequence of neurochemical and pathological changes predicted in Braak's model. Our results don't offer a scientifically sound validation of Braak's "dual-hit" hypothesis (which would require a prospective study of asymptomatic individuals periodically assessed regarding intestinal permeability and parkinsonism). However, if coupled with the results of Pan-Montojo's group, they could be viewed as favoring its biologic plausibility.

We were unable to determine whether the gastrointestinal permeability abnormalities preceded any of their reported symptoms (including gastrointestinal premotor complaints and parkinsonism). Bacterial overgrowth and infection with Helicobacter pylori have both been recognized to occur frequently in the setting of gastrointestinal dysmotility and to lead to deranged mucosal permeability, thus our findings could reflect a long term complication of the involvement of the enteric nervous system in the disease process.

Besides the limitations that stem from the exploratory nature of our study, it must be acknowledged that it was not powered to disclose associations between the permeability abnormalities and gastrointestinal symptoms. Nevertheless, several observations may be made from our results, as depicted in the Table. All individuals with abnormal permeability test results had gastrointestinal symptoms and, as a group, they had nonsignificantly higher scores on the gastrointestinal items of the NMS-Quest as compared to participants with normal permeability test results. The number, frequency and severity of gastrointestinal symptoms in a given participant, however, were not reliable predictors of whether that individual would have abnormal test results. Likewise, the study was not powered to disclose potential associations between the permeability abnormalities and prodromal gastrointestinal symptoms, the duration, severity of PD, nor intended to evaluate potentially confounding factors, such as the various antiparkinsonian treatments.

This study has shown that gastrointestinal permeability is increased in a significant proportion of unselected PD patients with minimal gastrointestinal symptoms, but the significance of this finding needs to be further evaluated.

\section{REFERENCES}

1. Abbott RD, Ross GW, White LR, et al. Environmental, life-style, and physical precursors of clinical Parkinson's disease: recent findings from the Honolulu-Asia Aging Study. J Neurol. 2003 Oct;250 Suppl 3:III30-9.

2. Kaye J, Gage H, Kimber A, et al. Excess burden of constipation in Parkinson's disease: a pilot study. Mov Disord. 2006 Aug;21(8): 1270-3.

3. Savica R, Carlin JM, Grossardt BR, et al. Medical records documentation of constipation preceding Parkinson disease: A case-control study. Neurology. 2009 Nov 24;73(21):1752-8.

4. Abbott RD, Ross GW, Petrovitch H, et al. Bowel movement frequency in late-life and incidental Lewy bodies. Mov Disord. 2007 Aug 15;22(11):1581-6.

5. Petrovitch H, Abbott RD, Ross GW, et al. Bowel movement frequency in late-life and substantia nigra neuron density at death. Mov Disord. 2009 Feb 15;24(3):371-6.
6. Hawkes $\mathrm{CH}$, Del Tredici K, Braak H. Parkinson's disease: a dualhit hypothesis. Neuropathol Appl Neurobiol. 2007 Dec;33(6): 599-614.

7. Lebouvier T, Chaumette T, Paillusson S, et al. The second brain and Parkinson's disease. Eur J Neurosci. 2009 Sep;30(5):735-41.

8. Jost WH. Gastrointestinal dysfunction in Parkinson's Disease. J Neurol Sci. 2010 Feb 15;289(1-2):69-73.

9. Teshima CW, Meddings JB. The measurement and clinical significance of intestinal permeability. Curr Gastroenterol Rep. 2008 Oct; 10(5):443-9.

10. Hughes AJ, Daniel SE, Kilford L, Lees AJ. Accuracy of clinical diagnosis of idiopathic Parkinson's disease: a clinicopathological study of 100 cases. J Neurol Neurosurg Psychiatry. 1992 Mar;55(3):181-4.

11. Tomlinson CL, Stowe R, Patel S, et al, Systematic review of levodopa dose equivalency reporting in .Parkinson's disease. Mov Disord. 2010 Nov 15;25(15):2649-53.

12. Chaudhuri KR, Martinez-Martin P, Schapira AH, et al. International multicenter pilot study of the first comprehensive self-completed nonmotor symptoms questionnaire for Parkinson's disease: the NMSQuest study. Mov Disord. 2006 Jul;21(7):916-23.

13. Goetz CG, Tilley BC, Shaftman SR, et al on behalf of the Movement Disorder Society UPDRS Revision Task Force. Movement Disorder Society-sponsored revision of the Unified Parkinson's Disease Rating Scale (MDS-UPDRS): scale presentation and clinimetric testing results. Mov Disord. 2008 Nov 15;23(15):2129-70.

14. Bushara KO. Neurologic presentation of celiac disease. Gastroenterology. 2005 Apr;128(4 Suppl 1):S92-7.

15. Vogelsang $H$, Schwarzenhofer $M$, Oberhuber G. Changes in gastrointestinal permeability in celiac disease. Dig Dis. 1998 Nov-Dec;16(6):333-6.

16. Edelblum KL, Turner JR. The tight junction in inflammatory disease: communication breakdown. Curr Opin Pharmacol. 2009 Dec;9(6):715-20.

17. Ohman L, Simrén M. Pathogenesis of IBS: role of inflammation, immunity and neuroimmune interactions. Nat Rev Gastroenterol Hepatol. 2010 Mar;7(3):163-73.

18. De Keyser F, Elewaut D, De Vos M, et al. Bowel inflammation and the spondyloarthropathies. Rheum Dis Clin North Am. 1998 Nov;24(4):785-813, ix-x

19. Feld JJ, Meddings J, Heathcote EJ. Abnormal intestinal permeability in primary biliary cirrhosis. Dig Dis Sci. 2006 Sep; 51(9):1607-13.

20. Pirlich M, Norman K, Lochs H, Bauditz J. Role of intestinal function in cachexia. Curr Opin Clin Nutr Metab Care. 2006 Sep;9(5):603-6.

21. Douek D. HIV disease progression: immune activation, microbes, and a leaky gut. Top HIV Med. 2007 Aug-Sep;15(4):114-7.

22. Liu H, Li W, Wang X, Li J, Yu W. Early gut mucosal dysfunction in patients with acute pancreatitis. Pancreas. 2008 Mar;36(2):192-6.

23. Sandek A, Rauchhaus M, Anker SD, von Haehling S. The emerging role of the gut in chronic heart failure. Curr Opin Clin Nutr Metab Care. 2008 Sep;11(5):632-9.

24. Vaarala O. Leaking gut in type 1 diabetes. Curr Opin Gastroenterol. 2008 Nov;24(6):701-6.

25. Miele L, Valenza V, La Torre G, et al. Increased intestinal permeability and tight junction alterations in nonalcoholic fatty liver disease. Hepatology. 2009 Jun;49(6):1877-87.

26. Davies KN, King D, Billington D, et al. Intestinal permeability and orocaecal transit time in elderly patients with Parkinson's disease. Postgrad Med J. 1996 Mar;72(845):164-7.

27. Goddard AF. Review article: factors influencing antibiotic transfer across the gastric mucosa. Aliment Pharmacol Ther. 1998 Dec; 12(12):1175-84.

28. Höglinger GU, Oertel WH, Hirsch EC. The rotenone model of parkinsonism--the five years inspection. J Neural Transm Suppl. 2006;(70):269-72.

29. Pan-Montojo F, Anichtchik O, Dening Y, et al. Progression of Parkinson's disease pathology is reproduced by intragastric administration of rotenone in mice. PLoS One. 2010 Jan 19;5(1): e8762. 